\title{
Critical Field of Spin Torque Oscillator with Perpendicularly Magnetized Free Layer
}

\author{
Tomohiro Taniguchi, Hiroko Arai, Sumito Tsunegi, Shingo Tamaru, Hitoshi Kubota, and Hiroshi Imamura \\ National Institute of Advanced Industrial Science and Technology (AIST), \\ Spintronics Research Center, \\ 1-1-1 Umezono, Tsukuba 305-8568, Japan
}

\begin{abstract}
The oscillation properties of a spin torque oscillator consisting of a perpendicularly magnetized free layer and an in-plane magnetized pinned layer are studied based on an analysis of the energy balance between spin torque and damping. The critical value of an external magnetic field applied normal to the film plane is found, below which the controllable range of the oscillation frequency by the current is suppressed. The value of the critical field depends on the magnetic anisotropy, the saturation magnetization, and the spin torque parameter.
\end{abstract}

The self-oscillation of the magnetization in a spin torque oscillator (STO) has been studied extensively because of its potential application to spintronics devices such as microwave generators and recording heads of high-density hard disk drives [1-10]. The self-oscillation of the magnetization is induced when the energy supply from the spin torque balances with the energy dissipation due to the damping [11]. Recently, it was found that the STO consisting of a magnetic tunnel junction (MTJ) with a perpendicularly magnetized free layer and an in-plane magnetized pinned layer [12 14] showed a large power $(\sim 0.5 \mu \mathrm{W})$ and a narrow linewidth $(\sim 50$ $\mathrm{MHz}$ [15], making a great advance toward the realization of STO devices.

Precise control of the oscillation frequency by the current is necessary for STO application. To this end, it is important to clarify the oscillation properties of STOs. Depending on the magnetization directions of the free and pinned layers, STO can be classified into four types. The self-oscillation of the STO was first observed in the in-plane magnetized system in 2003 [1]. An MTJ with an in-plane magnetized free layer and a perpendicularly magnetized pinned layer was also developed because a significant reduction in the switching current was expected [3, 16, 17]. An STO in which both the free and pinned layers were perpendicularly magnetized was theoretically studied because its axial symmetry made the analyses easy [18]. Contrary to these three types, the oscillation properties of STO with a perpendicularly magnetized free layer and an in-plane magnetized pinned layer remain unclear.

In this letter, we theoretically study the oscillation properties of an STO with a perpendicularly magnetized free layer and an in-plane magnetized free layer based on the analyses of the energy balance between spin torque and damping. We find that an external magnetic field applied normal to the film plane plays a key role in the self-oscillation of the magnetization. A critical value of the applied field exists below which the controllable range of the oscillation frequency by the current is suppressed. The value of the critical field depends on the perpendicular magnetic anisotropy, the saturation magnetization, and the spin torque parameter.

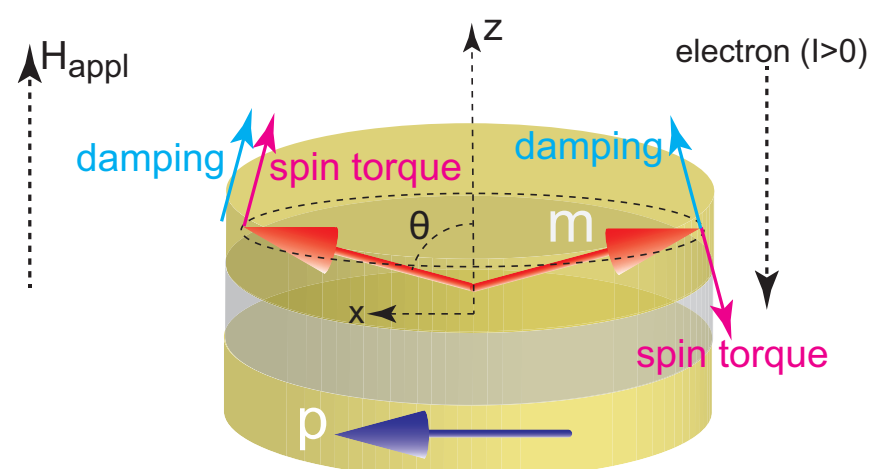

FIG. 1: Schematic view of the system, where $\mathbf{m}$ and $\mathbf{p}$ are the unit vectors pointing in the magnetization directions of the free and pinned layers, respectively. The tilted angle of the magnetization $\mathbf{m}$ from the $z$-axis is denoted as $\theta$. The arrows indicate the directions of spin torque and damping. The applied field is denoted as $H_{\text {appl }}$.

The system we consider is schematically shown in Fig. 1. where the unit vectors pointing in the magnetization directions of the free and pinned layers are denoted as $\mathbf{m}$ and $\mathbf{p}$, respectively. The $z$-axis is normal to the film plane while the $x$-axis is parallel to $\mathbf{p}$. The applied field, $H_{\text {appl }}$, is parallel to the $z$-axis. The current is denoted as $I$, where the positive current corresponds to the electrons flowing from the free layer to the pinned layer. The magnetic energy density of the free layer, $E=-M H_{\mathrm{appl}} m_{z}-\left[M\left(H_{\mathrm{K}}-4 \pi M\right) / 2\right] m_{z}^{2}$, consists of the Zeeman energy and the uniaxial anisotropy energy, where $M$ and $H_{\mathrm{K}}$ are the magnetization and the crystalline anisotropy along the $z$-axis, respectively. Because we are interested in the perpendicularly magnetized free layer, $H_{\mathrm{K}}$ should be larger than the demagnetization field $4 \pi M$. The energy density has two minima at $\mathbf{m}= \pm \mathbf{e}_{z}$. Throughout this letter, the initial state is assumed to be $\mathbf{m}=\mathbf{e}_{z}$. It should be noted that a trajectory with a constant $m_{z}=\cos \theta$ corresponds to the constant energy line of this system. The magnetization dynamics is described by the Landau-Lifshitz-Gilbert (LLG) equation [19 23],

$$
\frac{d \mathbf{m}}{d t}=-\gamma \mathbf{m} \times \mathbf{H}-\gamma H_{\mathrm{s}} \mathbf{m} \times(\mathbf{p} \times \mathbf{m})+\alpha \mathbf{m} \times \frac{d \mathbf{m}}{d t},
$$

where $\gamma$ and $\alpha$ are the gyromagnetic ratio and Gilbert 
damping constant, respectively. The magnetic field is defined as $\mathbf{H}=-\partial E /(\partial M \mathbf{m})$. The strength of the spin torque is

$$
H_{\mathrm{s}}=\frac{\hbar \eta I}{2 e M V(1+\lambda \mathbf{m} \cdot \mathbf{p})},
$$

where $V$ is the volume of the free layer. Two dimensionless parameters, $\eta$ and $\lambda$, whose ranges are $0<\eta<1$ and $-1<\lambda<1$, determine the magnitude of the spin polarization and the dependence of the spin torque strength on the relative angle between the magnetizations $\left(\cos ^{-1} \mathbf{m} \cdot \mathbf{p}\right)$, respectively. The relationships among $\eta, \lambda$, and other material parameters depend on the theoretical model: for example, in the ballistic transport theory in MTJs [21, 23], $\eta$ is proportional to the spin polarization of the density of state of the free layer and $\lambda=\eta^{2}$. The form of eq. (2) is common for spin torque in not only MTJs but also giant magnetoresistive (GMR) systems [22, 24]. In particular, $\lambda$ plays a key role in the magnetization dynamics of this system.

In the self-oscillation state, the energy supply by the spin torque balances with the energy dissipation due to the damping, and therefore, the magnetization precesses on the constant energy line. From eq. (1), the energy change due to the spin torque and the damping is described as $d E / d t=-M \mathbf{H} \cdot(d \mathbf{m} / d t)=\mathcal{W}_{\mathrm{s}}+\mathcal{W}_{\alpha}$, where

$$
\begin{gathered}
\mathcal{W}_{\mathrm{s}}=\frac{\gamma M H_{\mathrm{s}}}{1+\alpha^{2}}[\mathbf{p} \cdot \mathbf{H}-(\mathbf{m} \cdot \mathbf{p})(\mathbf{m} \cdot \mathbf{H})-\alpha \mathbf{p} \cdot(\mathbf{m} \times \mathbf{H})] \\
\mathcal{W}_{\alpha}=-\frac{\alpha \gamma M}{1+\alpha^{2}}\left[\mathbf{H}^{2}-(\mathbf{m} \cdot \mathbf{H})^{2}\right]
\end{gathered}
$$

are the work done by the spin torque and the dissipation due to the damping, respectively [25]. By assuming that the magnetization tilts from the $z$-axis with an angle $\theta=$ $\cos ^{-1} m_{z}$ and averaging $d E / d t$ over one precession period $\tau$, we found that the current $I(\theta)$ satisfying $\overline{d E / d t}=$ $(1 / \tau) \oint d t(d E / d t)=0$ is [26]

$$
\begin{aligned}
I(\theta)= & \frac{2 \alpha e \lambda M V}{\hbar \eta \cos \theta}\left(\frac{1}{\sqrt{1-\lambda^{2} \sin ^{2} \theta}}-1\right)^{-1} \\
& \times\left[H_{\mathrm{appl}}+\left(H_{\mathrm{K}}-4 \pi M\right) \cos \theta\right] \sin ^{2} \theta
\end{aligned}
$$

The oscillation frequency at the angle $\theta$ is $f(\theta)=1 / \tau=$ $\gamma\left[H_{\text {appl }}+\left(H_{\mathrm{K}}-4 \pi M\right) \cos \theta\right] /(2 \pi)$. The angle $\theta$ increases with increasing the current, which results red-shift of the oscillation frequency [27]. The critical current for precession is defined as $I_{\mathrm{c}}=\lim _{\theta \rightarrow 0} I(\theta)$, and is given by

$$
I_{\mathrm{c}}=\frac{4 \alpha e M V}{\hbar \eta \lambda}\left(H_{\mathrm{appl}}+H_{\mathrm{K}}-4 \pi M\right) .
$$

The critical current $I_{\mathrm{c}}$ diverges in the limit of $\lambda \rightarrow 0$ because when $H_{\mathrm{s}}$ is independent of the relative angle of the magnetization, and when the equilibrium direction of the magnetization is perpendicular to $\mathbf{p}$, the energy (a) $\mathrm{H}_{\mathrm{appl}}=0$

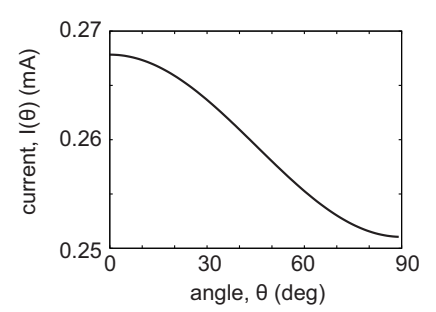

(b) $\mathrm{H}_{\mathrm{appl}}=10(\mathrm{Oe})$

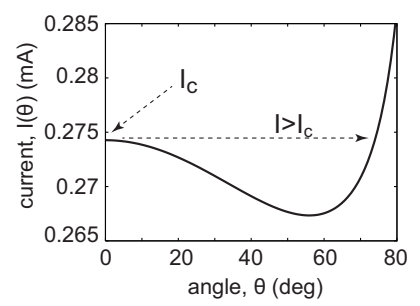

(c) $\mathrm{H}_{\text {appl }}=3(\mathrm{kOe})$

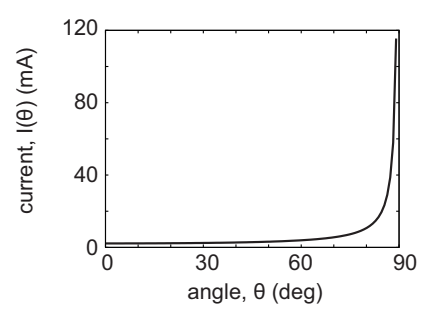

FIG. 2: Dependences of $I(\theta)$ [eq. (5)] on the tilted angle of the magnetization $\theta$ for (a) $H_{\text {appl }}=0$, (b) 10 , and (c) $3 \times 10^{3}$ Oe, respectively, where $\lim _{\theta \rightarrow 0} I(\theta)=I_{\mathrm{c}}$. The ranges of $\theta$ in (a) and (c) are $0 \leq \theta \leq 90^{\circ}$ while that in (b) is $0 \leq \theta \leq 80^{\circ}$ to emphasize the local minimum of $I(\theta)$.

supply from the spin torque over one precession period is zero, making it impossible for the spin torque to induce the magnetization dynamics.

Figures 2(a)-2(c) show the dependences of $I(\theta)$, eq. (5), on the tilted angle of the magnetization $\theta$ with $H_{\text {appl }}=0,10$, and $3 \times 10^{3}$ Oe, respectively. The values of the other parameters are $H_{\mathrm{K}}=18.6 \mathrm{kOe}, 4 \pi M=18.2$ $\mathrm{kOe}, V=\pi \times 60 \times 60 \times 2 \mathrm{~nm}^{3}, \eta=0.54, \lambda=\eta^{2}$, $\gamma=17.32 \mathrm{MHz} / \mathrm{Oe}$, and $\alpha=0.005$, which are estimated from the experiments [15, 28]. Depending on the value of the applied field, the dependence of $I(\theta)$ on $\theta$ is drastically changed, from which the following three distinguishable current dependences shown in Figs. 2(a)-2(c) are expected.

First, in the absence of the applied field, $I(\theta)$ monotonically decreases as the angle $\theta$ increases, and remains finite in the limit of $\theta \rightarrow \pi / 2$, as shown in Fig. 2(a). These indicate that, once the current magnitude reaches the critical current $I_{\mathrm{c}}$, the magnetization immediately moves to the film plane ( $x y$-plane) because $I(0<\theta<\pi / 2)<I_{\text {c }}$. Figure 3 shows the time evolutions of the component of $\mathbf{m}$ for $I=0.3 \mathrm{~mA}>I_{\mathrm{c}} \simeq 0.27 \mathrm{~mA}$. The magnetization reaches $\theta=\pi / 2$, and the dynamics stops at $\mathbf{m}=-\mathbf{e}_{x}$ because both the field torque and the spin torque, which are the first and second terms on the right-hand side of eq. (11), are zero at this point. Therefore, self-oscillation 


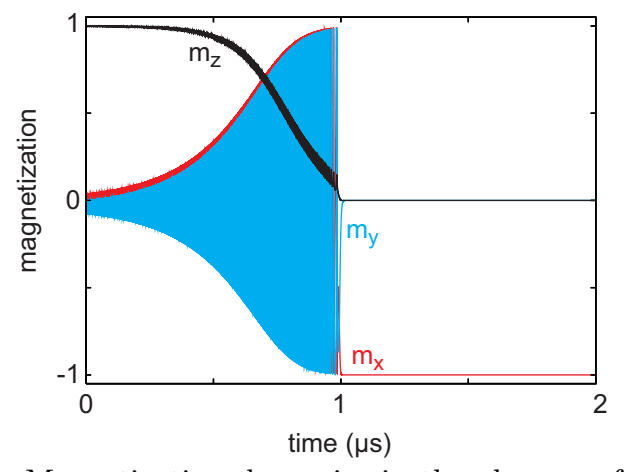

FIG. 3: Magnetization dynamics in the absence of the applied field, where the red, blue, and black lines correspond to $m_{x}, m_{y}$, and $m_{z}$, respectively. The red line $\left(m_{x}\right)$ below 1 $\mu$ s overlaps the blue line. The current magnitude is $0.3 \mathrm{~mA}$ $\left(>I_{\mathrm{c}} \simeq 0.27 \mathrm{~mA}\right)$.

cannot be realized in the absence of the applied field. It should be noted that since the spin torque prefers the anti-parallel alignment of the magnetizations for $I>0$, $\mathbf{m}$ stops at $-\mathbf{e}_{x}$, although all torques are also zero at $+\mathbf{e}_{x}$.

Second, when the magnetic field is smaller than a certain value $H_{\mathrm{c}}$, i.e., $0<H_{\text {appl }}<H_{\mathrm{c}}, I(\theta)$ shows a local minimum, as shown in Fig. 2(b). The theoretical formula and the value of $H_{\mathrm{c}}$ are derived below. In this intermediate region, when the current magnitude reaches $I_{\mathrm{c}}$, the magnetization moves to a certain angle $\theta_{0}$, which satisfies $I\left(\theta_{0}\right)=I_{\mathrm{c}}$. For example, in Fig. 2(b), $\theta_{0} \simeq 74^{\circ}$ for $H_{\mathrm{appl}}=10$ Oe. By increasing the current magnitude from $I_{\mathrm{c}}$, the tilted angle $\theta$ continuously increases from $\theta_{0}$. The self-oscillation can be realized with the frequency $f(\theta)$. It should be noted that, below $I_{\mathrm{c}}$, the power spectrum of the STO peaks at the ferromagnetic resonance $(\mathrm{FMR})$ frequency $f_{\mathrm{FMR}}=f(\theta=0)=$ $\gamma\left(H_{\text {appl }}+H_{\mathrm{K}}-4 \pi M\right) /(2 \pi)$ due to the mag-noise effect [15]. Since the tilted angle $\theta$ discontinuously changes at $I_{\mathrm{c}}$, the discontinuity of the oscillation frequency as a function of the current is expected, as shown in Fig. 4 (a).

Third, $I(\theta)$ monotonically increases as the current increases for the applied field satisfying $H_{\mathrm{c}}<H_{\text {appl }}$. In this case, the tilted angle of the magnetization continuously increases as the current increases from $I_{\mathrm{c}}$. Therefore, the oscillation frequency changes from $f_{\mathrm{FMR}}$ for the current

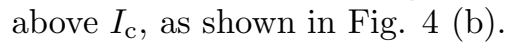

An important assumption used above is that the tilted angle of the magnetization, $\theta$, is constant during the precession. Based on this assumption, eq. (5) predicts that $\lim _{\theta \rightarrow \pi / 2} I(\theta)=\infty$ for $H_{\text {appl }}>0$, which means that the magnetization cannot reach the film plane. Then, the oscillation frequency saturates to $f(\theta=\pi / 2)=$ $\gamma H_{\text {appl }} /(2 \pi)$ in the large current limit. Also, the controllable range of the oscillation frequency for $H_{\mathrm{c}}<H_{\text {appl }}$ is $f(\theta=0)-f(\theta=\pi / 2)=\gamma\left(H_{\mathrm{K}}-4 \pi M\right) /(2 \pi)$, which depends on the perpendicular anisotropy only. Strictly speaking, however, the angle $\theta$ is not a constant during the precession because the directions of the spin torque (a)

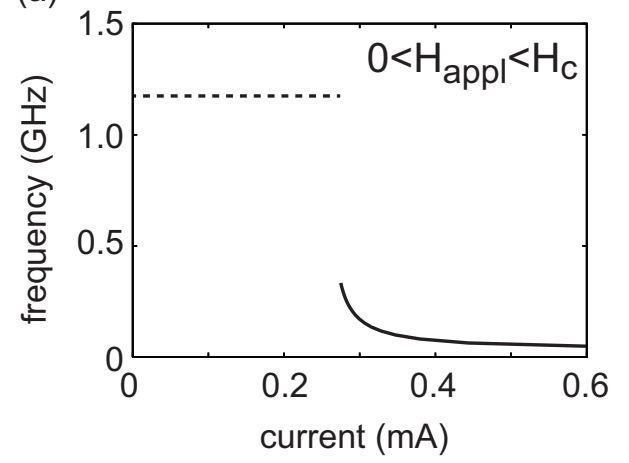

(b)

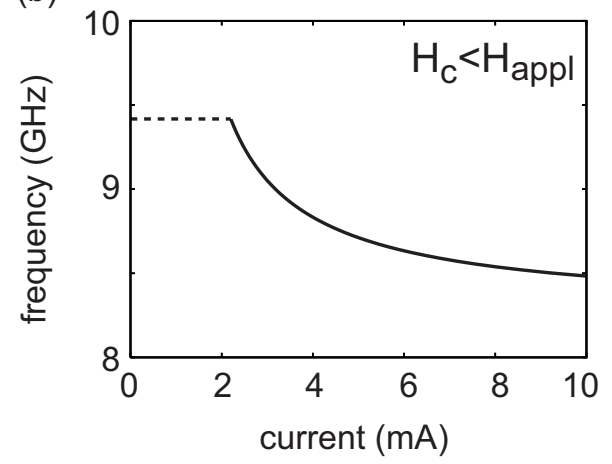

FIG. 4: The solid lines are the current dependences of the oscillation frequency in the self-oscillation state for (a) $0<$ $H_{\text {appl }}<H_{\mathrm{c}}$ and (b) $H_{\mathrm{c}}<H_{\text {appl }}$. The dashed lines are the FMR frequencies.

for $m_{x}>0$ and $m_{x}<0$ are opposite, as shown in Fig. 1. When $\theta$ becomes close to $\pi / 2$ by a large current, the magnetization can reach the film plane and stops its dynamics because the spin torque for $m_{x}<0$ moves the magnetization closer to the film plane. Therefore, in reality, the solid lines in Figs. 4(a) and 4(b) break at a certain current above which the self-oscillation cannot be realized. The magnitude of such current depends on the applied field magnitude. However, the investigation of such current or field magnitude requires a breakthrough of the constant $\theta$ assumption, and is beyond the scope of this letter.

The reason why the existence of the applied field determines whether the magnetization can reach $\theta=\pi / 2$ or not is as follows. The tilted angle of the magnetization $\theta$ is determined by the balance between the spin torque and the damping. In the absence of the applied field, the energy dissipation due to the damping, eq. (4), rapidly decreases as the angle $\theta$ increases, compared with the work done by spin torque, eq. (3), because $\mathcal{W}_{\alpha}$ is on the second order of the field $\mathbf{H}=\left(H_{\mathrm{K}}-4 \pi M\right) m_{z} \mathbf{e}_{z}$ while $\mathcal{W}_{\mathrm{s}}$ is on the first order of $\mathbf{H}$. Then, once the spin torque overcomes the damping at $\theta=0$, the energy supply from the spin torque is always larger than the energy dissipation due to the damping during $0<\theta<\pi / 2$. Therefore, the magnetization can reach $\theta=\pi / 2$. Because both the energy supply from the spin torque and the energy dissipation due to the damping are zero at 
$\theta=\pi / 2$, i.e., $\overline{d E(\theta=\pi / 2) / d t}=0$, the magnetization dynamics stops at $\theta=\pi / 2$. However, in the presence of the applied field, the damping can balance with the spin torque at $0<\theta<\pi / 2$ because of the presence of the constant term $H_{\mathrm{appl}}$ in the magnetic field $\mathbf{H}=\left[H_{\mathrm{appl}}+\left(H_{\mathrm{K}}-4 \pi M\right) m_{z}\right] \mathbf{e}_{z}$. Therefore, the selfoscillation of the magnetization with the angle $\theta$ can be realized. At $\theta=\pi / 2$, the direction of the spin torque is parallel to the film plane, which means that the work done by spin torque is zero. On the other hand, the energy dissipation due to the damping remains finite, i.e., $\overline{d E(\theta=\pi / 2) / d t}=-\alpha \gamma M H_{\text {appl }}^{2} /\left(1+\alpha^{2}\right)$. Therefore, the magnetization cannot reach $\theta=\pi / 2$.

The value of the critical field $H_{\mathrm{c}}$ can be determined by the condition in which $d I(\theta) / d \theta>0$ near $\theta \gtrsim 0$, and is given by

$$
H_{\mathrm{c}}=\frac{3 \lambda^{2}}{2-3 \lambda^{2}}\left(H_{\mathrm{K}}-4 \pi M\right),
$$

which is 59 Oe for the above parameters. We emphasize that $H_{\mathrm{c}}$ depends on the magnetic anisotropy and the spin torque parameter $\lambda$ only. As discussed above, when the applied field magnitude is larger than eq. (77), the controllable range of the oscillation frequency by the current is $\gamma\left(H_{\mathrm{K}}-4 \pi M\right) /(2 \pi)$. On the other hand, when $H_{\text {appl }}<H_{\mathrm{c}}$, the controllable range is suppressed because of the discontinuous change of the tilted angle of the magnetization.

Let us briefly discuss the relation between our previous work [15] and this work. Reference [15] experimentally investigated the current dependence of the oscillation frequency of STO. Because the applied field magnitude used in Ref. [15] (typically, $2 \mathrm{kOe}$ ) is much larger than $H_{\mathrm{c}}$, the continuous change of the oscillation frequency was observed, as shown in Fig. 3 (c) of Ref. [15].

At the end of this letter, let us mention that the sit- uation considered here is similar to the switching of the perpendicularly magnetized free layer by an in-plane polarized spin current injected by the spin-Hall effect [29]. Similar to the above discussion, in the spin-Hall system, the magnetization cannot cross over the film plane by the spin torque only, in principle. Therefore, to assist or prevent the switching, a magnetic field which has a component along the film plane was applied [29]. In the case of STO discussed in this letter, the field-like torque [30 32], which is neglected in the above calculation, plays a role of a torque due to a magnetic field along the film plane. Then, the field-like torque may changes the magnetization dynamics, especially in the zero-field limit. The investigation of the effect of the field-like torque will be an important work in future.

In conclusion, we studied the oscillation properties of the STO consisting of a perpendicularly magnetized free layer and an in-plane magnetized pinned layer by analyzing the energy balance between the spin torque and the damping. We found the existence of the critical value of the external magnetic field applied normal to the film plane, $H_{\mathrm{c}}$. When the applied field is below $H_{\mathrm{c}}$, the tilted angle of the magnetization discontinuously changes above the critical current. The controllable range of the oscillation frequency by the current is suppressed due to the discontinuity. Above $H_{\mathrm{c}}$, the controllable range of the oscillation frequency is $\gamma\left(H_{\mathrm{K}}-4 \pi M\right) /(2 \pi)$. The value of the critical field depends on the perpendicular magnetic anisotropy, the saturation magnetization, and the spin torque parameter $\lambda$.

The authors would like to acknowledge H. Naganuma, T. Yorozu, H. Maehara, A. Emura, M. Konoto, A. Fukushima, S. Okamoto, K. Kudo, H. Suto, T. Nagasawa, R. Sato, and M. Hayashi for their valuable discussions.
[1] S. I. Kiselev, J. C. Sankey, I. N. Krivorotov, N. C. Emley, R. J. Schoelkopf, R. A. Buhrman, and D. C. Ralph: Nature 425 (2003) 380.

[2] W. H. Rippard, M. R. Pufall, S. Kaka, S. E. Russek, and T. J. Silva: Phys. Rev. Lett. 92 (2004) 027201.

[3] D. Houssameddine, U. Ebels, B. Delaët, B. Rodmacq, I. Firastrau, F. Ponthenier, M. Brunet, C. Thirion, J.P. Michel, L. Prejbeanu-Buda, M.-C. Cyrille, O. Redon, and B. Dieny: Nat. Mater. 6 (2007) 447.

[4] A. M. Deac, A. Fukushima, H. Kubota, H. Maehara, Y. Suzuki, S. Yuasa, Y. Nagamine, K. Tsunekawa, D. D. Djayaprawira, and N. Watanabe: Nat. Phys. 4 (2008) 803.

[5] W. H. Rippard, A. M. Deac, M. R. Pufall, J. M. Shaw, M. W. Keller, and S. E. Russek: Phys. Rev. B 81 (2010) 014426 .

[6] K. Kudo, T. Nagasawa, K. Mizushima, H. Suto, and R. Sato: Appl. Phys. Express 3 (2010) 043002.

[7] H. Suto, T. Nagawasa, K. Kudo, K. Mizushima, and
R. Sato: Appl. Phys. Express 4 (2011) 013003.

[8] J. Sinha, M. Hayashi, Y. K. Takahashi, T. Taniguchi, M. Drapeko, S. Mitani, and K. Hono: Appl. Phys. Lett. 99 (2011) 162508.

[9] Z. Zeng, P. K. Amiri, I. Krivorotov, H. Zhao, G. Finocchio, J.-P. Wang, J. A. Katine, Y. Huai, J. Langer, K. Galatsis, K. L. Wang, and H. Jiang: ACS Nano 6 (2012) 6115.

[10] Z. Zeng, G. Finocchio, B. Zhang, P. K. Amiri, J. A. Katine, I. N. Krivorotov, Y. Huai, J. Langer, B. Azzerboni, K. L. Wang, and H. Jiang: Sci. Rep. 3 (2013) 1426.

[11] A. Slavin and V. Tiberkevich: IEEE. Trans. Magn. 45 (2009) 1875.

[12] S. Yakata, H. Kubota, Y. Suzuki, K. Yakushiji, A. Fukushima, S. Yuasa, and K. Ando: J. Appl. Phys. 105 (2009) 07D131.

[13] S. Ikeda, K. Miura, H. Yamamoto, K. Mizunuma, H. D. Gan, M. Endo, S. Kanai, J. Hayakawa, F. Matsukura, and H. Ohno: Nat. Mater. 9 (2010) 721. 
[14] H. Kubota, S. Ishibashi, T. Saruya, T. Nozaki, A. Fukushima, K. Yakushiji, K. Ando, Y. Suzuki, and S. Yuasa: J. Appl. Phys. 111 (2012) 07C723.

[15] H. Kubota, K. Yakushiji, A. Fukushima, S. Tamaru, M. Konoto, T. Nozaki, S. Ishibashi, T. Saruya, S. Yuasa, T. Taniguchi, H. Arai, and H. Imamura: Appl. Phys. Express 6 (2013) 103003.

[16] A. D. Kent, B. Ozyilmaz, and E. del Barco: Appl. Phys. Lett. 84 (2004) 3897.

[17] K. J. Lee, O. Redon, and B. Dieny: Appl. Phys. Lett. 86 (2005) 022505.

[18] T. J. Silva and M. W. Keller: IEEE Trans. Magn. 46 (2010) 3555.

[19] E. M. Lifshitz and L. P. Pitaevskii: Statistical Physics (part 2) (course of theoretical physics volume 9. Butterworth-Heinemann, Oxford, 1980) first ed., course of theoretical physics volume 9, Chap. 7.

[20] T. L. Gilbert: IEEE Trans. Magn. 40 (2004) 3443.

[21] J. C. Slonczewski: Phys. Rev. B 39 (1989) 6995.

[22] J. C. Slonczewski: J. Magn. Magn. Mater. 159 (1996) L1.

[23] J. C. Slonczewski: J. Magn. Magn. Mater. 247 (2002) 324.

[24] J. Xiao, A. Zangwill, and M. D. Stiles: Phys. Rev. B 70
(2004) 172405.

[25] T. Taniguchi, Y. Utsumi, M. Marthaler, D. S. Golubev, and H. Imamura: Phys. Rev. B 87 (2013) 054406.

[26] T. Taniguchi, H. Arai, H. Kubota and H. Imamura: accepted to IEEE. Trans. Magn.

[27] Blue-shift of the oscillation frequency can be expected when the crystalline anisotropy field $H_{\mathrm{K}}$ is smaller than the demagnetization field $4 \pi M$ and the applied field magnitude is larger than $4 \pi M-H_{\mathrm{K}}$.

[28] M. Konoto, H. Imamura, T. Taniguchi, K. Yakushiji, H. Kubota, A. Fukushima, K. Ando, and S. Yuasa: Appl. Phys. Express 6 (2013) 073002.

[29] L. Liu, O. J. Lee, T. J. Gudmundsen, D. C. Ralph, and R. A. Buhrman: Phys. Rev. Lett. 109 (2012) 096602.

[30] A. A. Tulapurkar, Y. Suzuki, A. Fukushima, H. Kubota, H. Maehara, K. Tsunekawa, D. D. Djayaprawira, N. Watanabe, and S. Yuasa: Nature 438 (2005) 339.

[31] H. Kubota, A. Fukushima, K. Yakushiji, T. Nagahama, S. Yuasa, K. Ando, H. Maehara, Y. Nagamine, K. Tsunekawa, D. D. Djayaprawira, N. Watanabe, and Y. Suzuki: Nat. Phys. 4 (2008) 37.

[32] J. C. Sankey, Y.-T. Cui, J. Z. Sun, J. C. Slonczewski, R. A. Buhrman, and D. C. Ralph: Nat. Phys. 4 (2008) 67. 